\title{
A Sequential Compressed Spectrum Sensing Algorithm against SSDH Attack in Cognitive Radio Networks
}

\author{
Zhuhua Hu, ${ }^{1,2}$ Yong Bai $\mathbb{D},{ }^{1,2}$ Lu Cao $\mathbb{D}^{1},{ }^{1}$ Mengxing Huang $\mathbb{D}^{1,2}$ and Mingshan Xie $\mathbb{D}^{1}$ \\ ${ }^{1}$ College of Information Science \& Technology, Hainan University, Haikou 570228, China \\ ${ }^{2}$ State Key Laboratory of Marine Resource Utilization in South China Sea, Hainan University, Haikou 570228, China \\ Correspondence should be addressed to Yong Bai; bai@hainu.edu.cn
}

Received 10 February 2017; Revised 11 August 2017; Accepted 1 November 2017; Published 2 January 2018

Academic Editor: George S. Tombras

Copyright (c) 2018 Zhuhua Hu et al. This is an open access article distributed under the Creative Commons Attribution License, which permits unrestricted use, distribution, and reproduction in any medium, provided the original work is properly cited.

\begin{abstract}
Spectrum sensing is one of the key technologies in wireless wideband communication. There are still challenges in respect of how to realize fast and robust wideband spectrum sensing technology. In this paper, a novel nonreconstructed sequential compressed wideband spectrum sensing algorithm (NSCWSS) is proposed. Firstly, the algorithm uses a sequential spectrum sensing method based on history memory and reputation to ensure the robustness of the algorithm. Secondly, the algorithm uses the strategy of compressed sensing without reconstruction, which thus ensures the sensing agility of the algorithm. The algorithm is simulated and analyzed by using the centralized cooperative sensing. The theoretical analysis and simulation results reveal that, under the condition of ensuring the certain detection probability, the proposed algorithm effectively reduces complex computation of signal reconstruction, significantly reducing the wideband spectrum sampling rate. At the same time, in the cognitive wideband communication scenarios, the algorithm also achieves a better defense against the SSDF attack in spectrum sensing.
\end{abstract}

\section{Introduction}

Cognitive radio (CR) technology can increase the efficiency of spectrum utilization for wideband wireless communications [1]. Aiming at detecting spectrum holes, spectrum sensing is the precondition for the implementation of CR [2].

To detect the spectrum holes more effectively over a wide bandwidth in Cognitive Radio Network (CRN), traditional wideband spectrum sensing acquires the wideband signals with a high-speed analog-to-digital converter (ADC) and then uses digital signal processing techniques to detect spectral opportunities. However, it is very often limited by the capability of ADC hardware and unable to meet highspeed sampling rate stated in Nyquist sampling theorem for wideband spectrum sensing [3]. An effective solution to address the challenge is spectrum sensing technology based on the compressed sensing (or called compressed sampling, CS) theory. CS can maintain the structure and information of the original sparse signal far below the Nyquist sampling rate. There have been many research achievements in this field. Tian and Giannakis applied the CS to the research of wideband spectrum sensing and verified its effectiveness [4]. Collaborative spectrum sensing from sparse observations in CRNs is studied in [5] by applying matrix completion and joint sparsity recovery to reduce sensing and transmission requirements. In order to reduce computational cost, spectrum holes can be obtained by means of partial reconstruction. Hong [6] presented a method of detecting the primary users (PUs) based on Bayesian compressed sensing, which can estimate important parameters of the primary user's signal directly from compressed sampling without the need for complete reconstruction. Thus it greatly reduces the computational complexity, but it still needs some parameters' distribution information of the original sparse signal. Actually, partial and complete signal reconstruction may not be required in many spectrum sensing applications. A method was proposed in [7] for nonreconstruction compressed detection of random signal under the maximum likelihood criterion. A new method for blind detection of signals by using nonreconstruction compressed sampling without prior knowledge was proposed in [8].

In compressive observation, the redundant observation is usually contained. In order to effectively reduce the number 
of observations, the sequential theory was introduced into the wideband spectrum sensing, and sequential compressive spectrum sensing algorithm was proposed in $[9,10]$. The algorithm can make the compressive ratio adjusted adaptively according to the signal sparsity, which can reduce the sampling number, but this method still needs to reconstruct the original signal. In [11], according to the maximum interference endured for PU, the optimal false-alarm probability is set, which can obtain the largest throughput for second users (SUs) by improving the threshold method. However, the detected signal is still the deterministic signal, whose sparsity is known in [9-11]. In [12], nonreconstruction compressed sampling method combined with sequential testing for random signals was presented. In [12], only AWGN (Additive White Gaussian Noise) channel is considered, and the robustness of the algorithm was not guaranteed with malicious users (MUs) under the SSDF (Spectrum Sensing Data Falsification) attack [13].

When a priori knowledge of signal and sparsity is unknown, in order to agilely and robustly find out the spectrum holes not occupied by PUs, this paper presents an enhanced novel nonreconstructed sequential compressed wideband spectrum sensing (NSCWSS) algorithm. Firstly, a weighted sequential spectrum sensing method based on history memory model is designed. The method assumes that, in collaborative sensing environment, the initial credibility value of each secondary user is the same. After the end of each sensing, the value of credibility of corresponding users will be updated. Then the sensing results of secondary user are assigned with different weights corresponding to its credibility and history accuracy in the final fusion, which can improve the antijamming ability of the algorithm to launch SSDF attacks. In addition, the algorithm uses a compression sensing method without reconstruction, which combined with the theory of sequential detection; thus it can ensure the least average computation for signal detection.

\section{The Process of Nonreconstructed Compressed Detection}

In Cognitive Radio Network, SUs need to judge whether PUs exist or not for dynamic access without affecting the normal communications of the PUs. The compressed detection model can be expressed as

$$
y=\Phi x
$$

Under the fading channel, Corresponding to the cases that the PU does not exist or exist, a binary hypothesis testing model can be established as follows:

$$
\begin{aligned}
& H_{0}: y=\Phi \omega_{S L}, \\
& H_{1}: y=\Phi\left(h_{S L i} s+\omega_{S L}\right),
\end{aligned}
$$

where $s=[s(0), \ldots, s(n-1)]^{T}$ is the sequence of samples of random sparse signals, $h_{S L i}$ is the channel fading gain of the $i$ th sensing link, and $\omega_{S L}=\left[\omega_{S L}(0), \ldots, \omega_{S L}(n-1)\right]^{T}$ is the sequence of the samples of noise of the sensing link, which is independent and identically distributed (i.i.d) with one-sided power spectral density $N_{0}$. Here, we assume that $s$ and $\omega_{S L}$ are independent of each other; $n$ is the sampling number, and $\Phi$ is the observation matrix with dimension $m \times n$. Normally, $m=O(K \log (n / K))$ is required, where $K$ is the signal sparsity.

Considering fading channels, Rayleigh and Rician distributions do, in many cases, model the envelope of the signal through the fading channel very well. However, in the actual wireless communication environments, it is found that the Nakagami distribution provides better matching degree with the actual test [14]. In comparison with the Rician distribution, the Nakagami distribution does not need to be assumed in terms of direct conditions. Thus, the sensing links are assumed to be subject to a Nakagami- $m$ fading; then the received power $Y_{S L i}=v_{i}(t)^{2}$ of $i$ th CR user obeys a Gamma distribution. The probability density function (PDF) of $Y_{S L i}$ is given by [14-17]

$$
\begin{aligned}
& f_{Y_{S L i}}=\left(\frac{m_{i}}{\sigma_{Y_{S L i}}}\right)^{m_{i}} \frac{Y_{S L i}^{m_{i}-1}}{\Gamma\left(m_{i}\right)} \exp \left(-\frac{m_{i}}{\sigma_{Y_{S L i}}} Y_{S L i}\right) \\
& Y_{S L i} \geq 0,
\end{aligned}
$$

where $m_{i} \geq 1 / 2$ denotes the severity of fading, $\Gamma(\cdot)$ is the Gamma function, $\sigma_{Y_{S L i}}=E\left[Y_{S L i}\right]$ is the local-mean power of $v_{i}(t)$, and $v_{i}(t)=h_{S L i} s+\omega_{S L}$ denotes the received instantaneous signal level. As $m_{i}$ increases, the level of fading decreases. When $m_{i}=1 / 2$ or $m_{i}=1$, the sensing channel is subject to one-sided Gaussian fading and the Rayleigh fading, respectively. When $m_{i}>1$, the Nakagami fading can be approximately equivalent to the Rician fading. $\sigma_{Y_{S L i}}$ is given by [16]

$$
E\left[Y_{S L i}\right]= \begin{cases}\sigma_{Y_{S L i 0}}=N_{0} & H_{0} \\ \sigma_{Y_{S L i 1}}=E_{i}+N_{0}=N_{0}\left(1+\bar{\gamma}_{S L i}\right) & H_{1},\end{cases}
$$

where $\bar{\gamma}_{S L i}=E_{i} / N_{0}$ is the average SNR of the $i$ th sensing channel and $E_{i}=E\left[\left(h_{S L i} s\right)^{2}\right]$ is the expected local-mean power of the PUs under $H_{1}$ assumption.

In the nonreconstruction approach we can test the two assumptions in (2) by directly processing the observation vector $y=[y(0), \ldots, y(m-1)]^{T}$. Here, $m$ denotes the number of observations, and we assume that each observation is statistically independent of each other. Then, with $H_{0}$ assumption, the observation sequence $y$ is Gauss random vector with mean 0 and variance $N_{0} \Phi \Phi^{T}$. While the observation sequence $y$ is Gauss random vector under $H_{1}$ condition, its mean is $E\left(\Phi h_{S L i} s\right)$, and the variance is $\left(N_{0}\left(1+\bar{\gamma}_{S L i}\right)\right) \Phi \Phi^{T}$.

According to the Neyman-Pearson theorem [18], given a false-alarm probability $P_{f}=\alpha$, the detection probability $P_{d}$ is maximized with the test

$$
\Lambda(x)=\frac{p\left(y, H_{1}\right)}{p\left(y, H_{0}\right)}>\zeta .
$$

The threshold $\zeta$ is determined by

$$
P_{f}=\int_{\{y: \Delta(y)>\zeta\}} p\left(y \mid H_{0}\right) d y=\alpha .
$$


Putting the probability density function of $y$ into (5) and taking the logarithmic transformation [3], we can get

$$
\begin{aligned}
y^{T}\left(\Phi \Phi^{T}\right)^{-1} y & >2 \frac{N_{0}\left(N_{0}+E_{i}\right)}{E_{i}} \ln \left[\zeta \frac{\left(N_{0}+E_{i}\right)^{m / 2}}{N_{0}{ }^{m}}\right] \\
:=\eta . &
\end{aligned}
$$

Thus, the detection statistic for random signal based on nonreconstructed compressed sampling is expressed as

$$
t=y^{T}\left(\Phi \Phi^{T}\right)^{-1} y .
$$

Formula (8) contains all the information needed for signal compressed detection. In addition, if $\Phi=I$, it shows no compression capacity. That is to say, it is converted into the convertional energy-detection based spectrum sensing. The detection statistics $t$ follow $\chi_{m}^{2}$ distribution [12]. Then we can get

$$
\begin{aligned}
P_{f} & =\operatorname{Pr}\left\{t>\eta ; H_{0}\right\}=\operatorname{Pr}\left\{\frac{t}{N_{0}}>\frac{\eta}{N_{0}} ; H_{0}\right\} \\
& =Q_{\chi_{m}^{2}}\left(\frac{\eta}{N_{0}}\right)=\alpha, \\
\eta & =N_{0} Q_{\chi_{m}^{2}}^{-1}(\alpha), \\
P_{d} & =\operatorname{Pr}\left\{t>\eta ; H_{1}\right\}=\operatorname{Pr}\left\{\frac{t}{N_{0}+E_{i}}>\frac{\eta}{N_{0}+E_{i}}\right\} \\
& =Q_{\chi_{m}^{2}}\left(\frac{\eta}{N_{0}+E_{i}}\right),
\end{aligned}
$$

where the right tailed distribution of the random variables in $\chi_{m}^{2}$ distribution is $Q_{\chi_{m}^{2}}(x)=\int_{x}^{\infty} p(t) d t$.

Further, to simplify calculation, by using approximate expression method of $\chi_{m}^{2}$ distribution, we can get

$$
P_{f}=Q_{\chi_{m}^{2}}\left(\frac{\eta}{N_{0}}\right) \approx Q\left(\frac{\eta / N_{0}-m}{\sqrt{2 m}}\right) .
$$

From the above analysis, the relation equation $P_{d}(\alpha)$ between $P_{d}$ and $P_{f}$ can be given by

$$
P_{d}(\alpha)=Q\left(\frac{Q^{-1}(\alpha)-\left(E_{i} / N_{0}\right) \sqrt{m / 2}}{E_{i} / N_{0}+1}\right) .
$$

In (13), $Q(z)=\int_{z}^{\infty} \exp \left(-u^{2} / 2\right) d u$.

$\bar{\gamma}_{S L i}=E_{i} / N_{0}$ is the average SNR related to the $i$ th sensing channel, and then

$$
P_{d}(\alpha)=Q\left(\frac{Q^{-1}(\alpha)-\bar{\gamma}_{S L i} \sqrt{m / 2}}{\bar{\gamma}_{S L i}+1}\right) .
$$

Obviously, Because the $Q(\cdot)$ function is strictly monotonically decreasing, $P_{d}$ will increase with the increase of SNR $\bar{\gamma}_{S L i}$ and observation number $m$ under given $P_{f}$. From (9), the theoretical observation number $m$ required by the compressed detection algorithm can be given as

$$
m=2\left(\frac{N_{0} Q^{-1}(\alpha)-Q^{-1}\left(P_{d}\right)\left(N_{0}+E_{i}\right)}{E_{i}}\right)^{2} .
$$

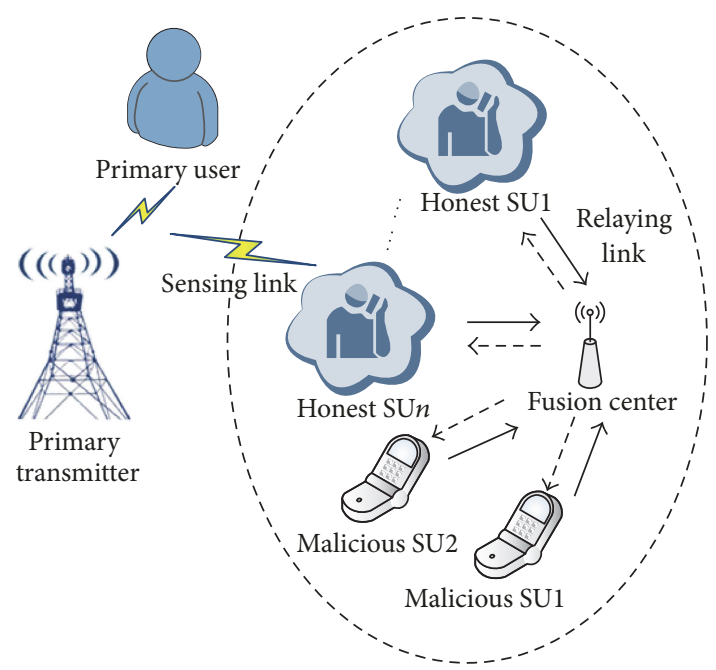

$\longrightarrow$ Sensing report

$-\rightarrow$ Decision feedback

Figure 1: System network model for centralized collaborative spectrum sensing with SSDF attack.

\section{System Modeling}

Centralized collaborative spectrum sensing can be seen as a series of processes of voting, decision, and fusion of binary decisions. Such a system is mainly composed of PU, SUs, malicious users, and fusion center (FC). The system model is shown in Figure 1.

In centralized collaborative spectrum sensing, the final result is obtained by the centralized fusion of the sensed information from each secondary user. Cognitive users and primary users coexist. We adopt the detect-forward (DF) relaying strategy between the SU and FC. All honest cognitive users need to submit their local-decsion, according to their independent observations, to the fusion center within a sampling period, respectively, meanwhile the SSDF attackers probably send false spectrum sensing reports, trying to cause a wrong global decision about spectrum availability at the FC [19]. The fusion center can determine the presence of primary user based on all collected local-decsions from SUs. On the basis of sequential sensing theory, when SU cannot make a decision at the $\mathrm{CR}$ node, an additional observation should be added.

In reality, it is difficult to predict the sparsity of the signals. When the predetermined value of the signal sparsity is larger than the actual one, it is inevitable to produce redundant observation sequence. These extra observation sequences increase the number of unnecessary compression measurements without improving the detection performance. On the other hand, if the predetermined value of the signal sparsity is set too low, the effective sample values of observation sequence are not enough, and it will be difficult to meet the requirements of sensing accuracy. Therefore, this paper adopts nonreconstruction method for spectrum sensing. A single SU detects signal based on sequential compressive sensing [9]. One row of $1 \times N$ vector $\Phi_{i}(i=1,2, \ldots)$ is 


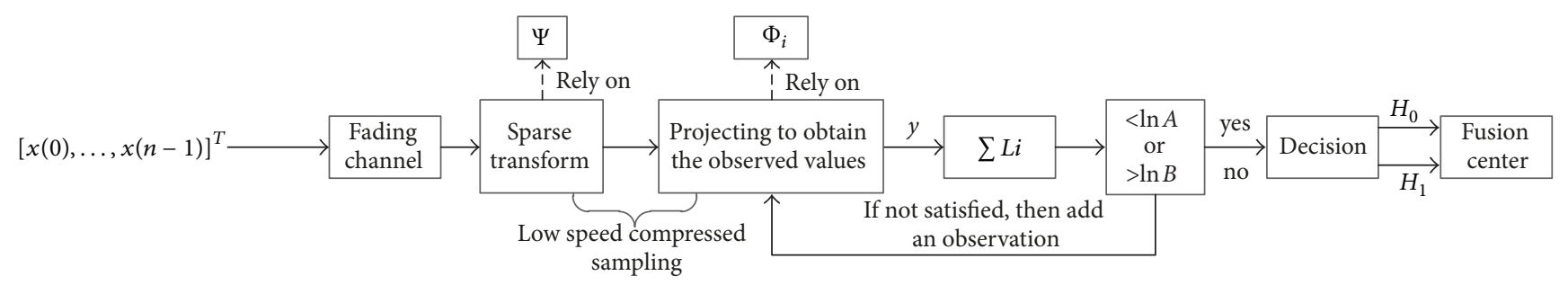

FIGURE 2: Spectrum sensing based on nonreconstructed sequential compression.

selected from the original observation matrix $\Phi$. SUs collect a fixed number of data $x=[x(0, \ldots, x(n-1))]^{Y}$ and project on the observation vector $\Phi_{i}$ to get low dimensional observation value $y_{i}$. Then, the likelihood ratio calculation, denoted as $L i$, is calculated and it is compared with the thresholds $\ln (A)$ and $\ln (B)$ and then sends feedback of the final results to the node. If we can make the decision with $\Phi_{i}$, then the test is over; otherwise, the observation vector $\Phi_{i+1}$ needs to be selected and $x$ is projected onto it. Then joint judgment is made together with previous observed values. Such a process is repeated until the decision can be made. In Figure 2, $\Psi$ is the sparse transformation matrix.

As mentioned in the Introduction, an effective solution for the technological challenge of ADC hardware is the wideband spectrum sensing technique based on compressed sensing theory. In fact, users just want to extract useful information from the observation sequence or filter out the information that they are not interested in subsequent processing. With respect to the sensing efficiency, it is not wise to completely reconstruct the original signal by using compressed sampling data.

The sequential nonreconstructed compressed sensing procedure, based on single CR node detection, is shown in Figure 2. The thresholds $A$ and $B$ are determined by $P_{d}$ and $P_{f}$, respectively, as

$$
\begin{aligned}
A & =\frac{1-P_{d}}{1-P_{f}}, \\
B & =\frac{P_{d}}{P_{f}} .
\end{aligned}
$$

From the above, we can rewrite (8) and get

$$
t_{i}=y_{i}^{T}\left(\Phi_{i} \Phi_{i}^{T}\right)^{-1} y_{i} .
$$

For the $k$ th CR user, we get a sampling sequence $X_{k}=$ $\left[x_{k}(0), \ldots, x_{k}(n-1)\right]^{T}, k=1,2, \ldots, N$, within the sampling period $T$. Then the data received at the $N$ CR nodes can be expressed in the matrix $\kappa=\left[X_{1}^{T}, \ldots, X_{N}^{T}\right]^{T}$. After the projection transformation between $\kappa$ and $\Phi_{i}$ is carried out, we can obtain $y_{i}=\left[y_{1 i}, \ldots, y_{N i}\right]^{T}$.

Putting each element of $y$ into (17), we can obtain the test value

$$
t_{i}=\left[t_{1 i}, \ldots, t_{k i}, \ldots, t_{N i}\right]^{T} \quad k=1,2, \ldots, N
$$

Obviously, if we take the $k$ th CR user as an example, $t_{k i}$ satisfies the $\chi^{2}$ distribution with the degree of freedom 1 [12].

$$
\begin{array}{r}
H_{1}: \frac{t_{k i}}{\left(N_{0}+E_{i}\right) \Phi_{i} \Phi_{i}^{T}} \sim \chi^{2}, \\
H_{0}: \frac{t_{k i}}{N_{0} \Phi_{i} \Phi_{i}^{T}} \sim \chi^{2} .
\end{array}
$$

Furthermore, we can obtain the following formula by means of the log-likelihood ratio:

$$
\begin{aligned}
\sum_{m=1}^{M} L i & =\sum_{m=1}^{M} \ln \frac{P_{m}\left(t_{k i} \mid H_{1}\right)}{P_{m}\left(t_{k i} \mid H_{0}\right)} \\
& =\sum_{m=1}^{M}\left(\ln \frac{P_{m}\left(t_{k i} \mid H_{1}\right)}{P_{m}\left(t_{k i} \mid H_{0}\right)}\right) \\
& =\sum_{m=1}^{M}\left(\ln \left(\frac{N_{0}}{E_{i}+N_{0}}\right)^{1 / 2}+\frac{t_{k i} E_{i}}{2 N_{0}\left(N_{0}+E_{i}\right)}\right)_{m},
\end{aligned}
$$

where $M$ is the number of sequential observations. $\sum L i$ denotes the sum of the current value of $L i$ and the value of previous likelihood ratio. Comparing $\sum L i$ with $\ln (A)$ and $\ln (B)$ continually until the decision can be made, then we can also get the theoretical value of the total average number of observations:

$$
\begin{aligned}
E[M]= & \frac{\left(\sum_{n=1}^{N} E_{n}[M]\right)}{N} \\
= & \frac{\sum_{n=1}^{N}\left(P\left(H_{1}\right)\left(\left(P_{d} \ln B+\left(1-P_{d}\right) \ln A\right) /\left(\ln \sqrt{N_{0} /\left(E_{i}+N_{0}\right)}+E_{i} / 2 N_{0}\right)\right)\right)_{n}}{N} \\
& +\frac{\sum_{n=1}^{N}\left(P\left(H_{0}\right)\left(\left(P_{f} \ln B+\left(1-P_{f}\right) \ln A\right) /\left(\ln \sqrt{N_{0} /\left(E_{i}+N_{0}\right)}+E_{i} / 2\left(N_{0}+E_{i}\right)\right)\right)\right)_{n}}{N} .
\end{aligned}
$$


From (21), we can know that the number of observations is closely related to the signal-to-noise ratio.

\section{NSCWSS Algorithm}

\subsection{Traditional Sequential Detection [9]}

$$
S_{n}=\prod_{i=0}^{n} \frac{P\left(S_{i} \mid H_{1}\right)}{P\left(S_{i} \mid H_{0}\right)},
$$

where the sampling value $n$ is arbitrary value of the number of all collaborative nodes and we take the double threshold detection method for detection. With given $P_{f}$ and $P_{m d}$, the two thresholds can be expressed as

$$
\begin{aligned}
& \xi_{0}=\frac{P_{f}}{1-P_{m d}}, \\
& \xi_{1}=\frac{1-P_{f}}{P_{m d}} .
\end{aligned}
$$

The rules of double threshold decision are as follows:

$$
\begin{aligned}
& S_{n} \geq \xi_{1} \longrightarrow H_{1}, \\
& S_{n} \leq \xi_{0} \longrightarrow H_{0}, \\
& \xi_{1} \geq S_{n} \geq \xi_{0} \longrightarrow \text { continue. }
\end{aligned}
$$

4.2. Algorithm Idea. In traditional sequential detection, each data fusion does not need to traverse all the CR user's sensing results, which improves the sensing speed. Nevertheless, the deficiency is that it treats all the nodes equivalently, which affects the effectiveness and accuracy of each sensing for different CR nodes.

In the process of centralized collaborative spectrum sensing detection, there might be a secondary user simulating the authorized signal to send false information to gain a higher access authority, which makes the fusion center to make a false judgment and thus seriously affects the efficiency and accuracy of overall sensing. In order to ensure that the collaborative spectrum sensing under SSDF attacks to have a high degree detection accuracy, the aggregation center needs to identify and try to eliminate the interference of false spectrum detection information sent by those malicious users. In addition, the occurrences of these disturbances are random and uncertain, so we need a better data fusion scheme. A weighted sequential probability ratio test (WSPRT) is presented in [20]. An improved WSPRT algorithm with advanced weight sequential log-likelihood ratio detection (AWSPRT) is presented in [21]. Those proposed methods apply information of trust degree to data fusion, and the fusion center distinguishes the credibility of spectrum sensing results by trust value, which can be against SSDF attack to a certain extent. However, because these two algorithms have no combination with compressed sampling and the computational complexity is high, they are hard to be used for wideband spectrum sensing.
For the problems mentioned above, this paper proposes a robust weighted sequential spectrum sensing algorithm based on history information and compressed nonreconstruction sampling technique. The proposed algorithm assigns weights to SUs by building weighted vector $W$. Meanwhile, the fusion center will efficiently record each sensing decision result. When there exists malicious signal, FC gives them lower weights to decrease the trust degree of sensor nodes and carries on the record. Therefore, the interference of the malicious nodes on the whole sensing network can be reduced. Another benefit, by the introduction of the nonreconstructed compressed sensing approach, is that our proposed algorithm (NSCWSS) can greatly reduce the sensing time by the lower computing complexity. Structure description of the NSCWSS algorithm, based on DF strategy, is as shown in Figure 3.

In FC, each sensing result of all SUs is recorded by using a struct. All record nodes are stored by using data struct of block linked list, which is described as Figure 4. Struct fields are all initially set to 0 . Figure 4 records $N$ times' spectrum sensing results for each secondary user. In Figure 4, $b_{j i}$ denotes difference between decision result of $\mathrm{SU}_{j}$ and $\mathrm{FC}$ in the $i$ th time, and $l_{j i}$ is the concept of correct rate, which is the ratio of the times that the spectrum sensing decision results of $\mathrm{SU}_{j}$ are the same as the final decision of the fusion center to the first $i$ times.

The value of $b_{j i}$ and $l_{j i}$ is calculated as follows:

$$
\begin{aligned}
& b_{j i}= \begin{cases}0, & H_{j i}=H_{\mathrm{FC}} \\
1, & H_{j i} \neq H_{\mathrm{FC}}\end{cases} \\
& l_{j i}=1-\frac{\sum_{m=1}^{i} b_{j m}}{i} .
\end{aligned}
$$

In [20], if the user is judged to be a malicious user, its weight is set to 0 . That is, the data of malicious users is abandoned and will not participate in the process of data fusion. When there are many malicious users, the data sent to the fusion center from secondary users may not reach decision condition. Then the detection performance of the system will decline, which wastes information resources. If we give a negative value to the data from less trusted users, this will effectively reduce the waste of the information resources.

We set the reputation vector to $R=\left[r_{1}, r_{2}, \ldots, r_{i}, \ldots\right.$, $\left.r_{M-1}, r_{M}\right]^{T}$, and initial value of elements is set to 0 , which is corresponding to the reputation value of each CR user. After each round of sensing, the corresponding user's reputation value will be updated. In the real environment, once there is a malicious user, detection probability will drop sharply. So, the cumulative rate of accuracy is the $2 / 3$ of attenuation rate. The update of credibility is performed as follows:

$$
\begin{aligned}
& r_{j}=r_{j}+1 \quad H_{j i}=H_{\mathrm{FC}}, \\
& r_{j}=r_{j}-1.5 \quad H_{j i} \neq H_{\mathrm{FC}} .
\end{aligned}
$$

Here we assume that the sensing weight of each CR node is $w_{i}$, and its initial value is set to 1 . Constant $-g$ is as the lower limit of credibility. $g$ is usually assigned the value 5 . 


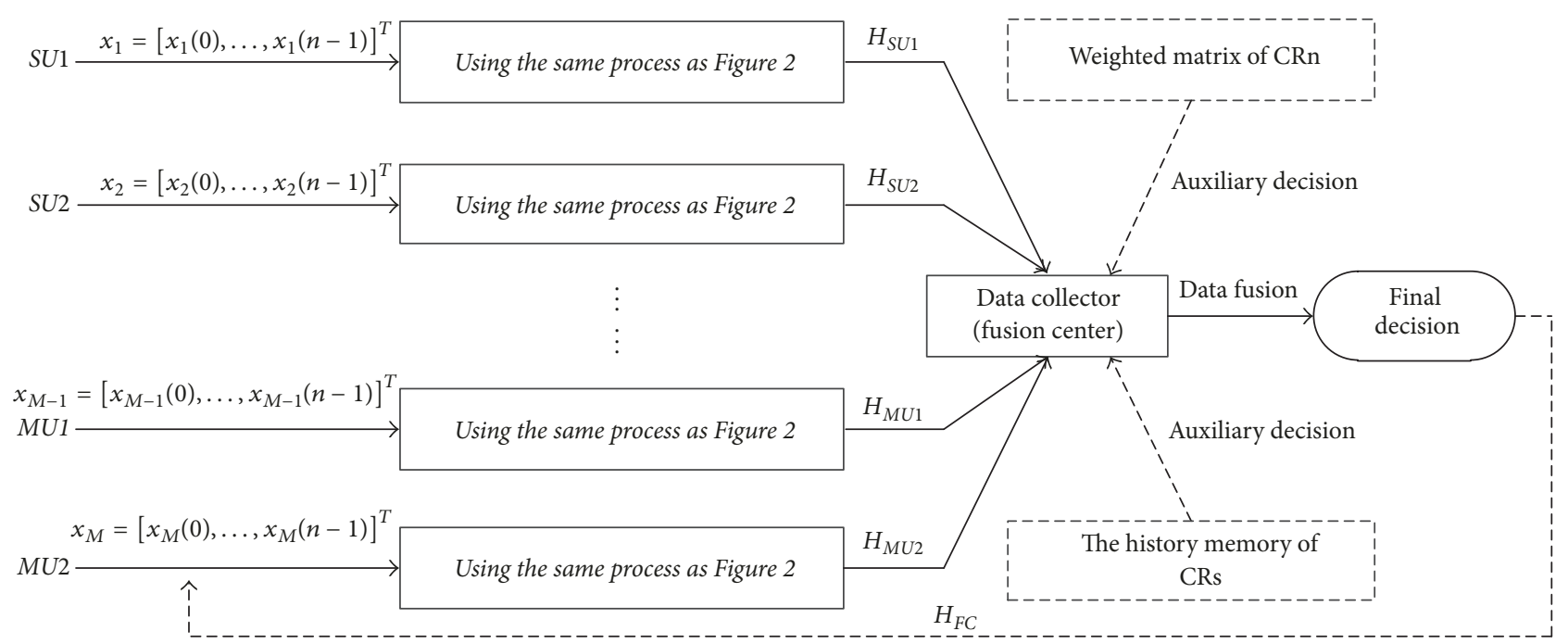

FIGURE 3: The flow chart of NSCWSS algorithm.

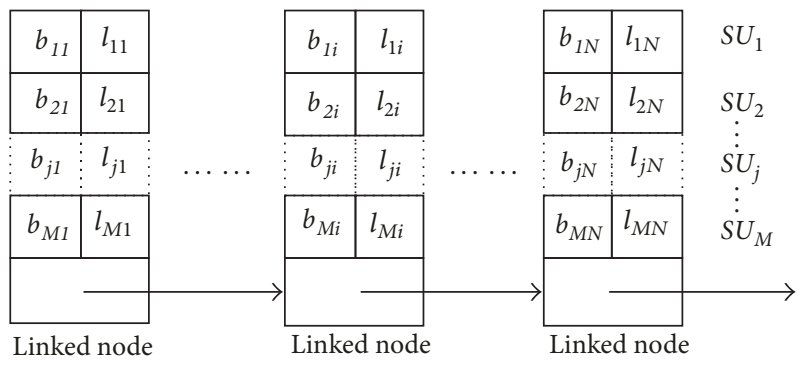

FIGURE 4: History memory model.

Then we set weighted coefficient to $W_{i}=\left[w_{1 i}, w_{2 i}, \ldots\right.$, $\left.w_{j i}, \ldots, w_{(M-1) i}, w_{M i}\right]^{T}$ in the $i$ th sensing cycle:

$$
w_{j i}=f\left(r_{j i}\right) \begin{cases}\frac{r_{j i} \times l_{j i}}{\max \left(r_{i}\right)+g}, & r_{j i} \leq-g \\ \frac{r_{j i} \times l_{j i}+g}{\max \left(r_{i}\right)+g}, & r_{j i}>-g .\end{cases}
$$

Normalization of $w_{j i}$ is required in applications. Then the new decision variables can be expressed as

$$
S_{M}=\prod_{i=0}^{M}\left(\frac{P\left[S_{i} \mid H_{1}\right]}{P\left[S_{i} \mid H_{0}\right]}\right)^{w_{j i}}
$$

The NSCWSS algorithm compares the result $S_{M}$ with the two threshold values of $\xi_{0}$ and $\xi_{1}$ to get the final decision results. The fusion center needs to analyze the reliability degree of each user's information and then compares the test results of secondary user with the final fusion results to evaluate the accuracy of CR user's detection and improve the capability of anti-interference of system on SSDF attack. Compared to the previous algorithms, our proposed algorithm has strong robustness in the situation of collaborative spectrum sensing where there are malicious users. Thus it can also effectively resist the SSDF attack and improves the detection probability of system and significantly reduces the wrong judgment of the whole system.

\section{Simulation Results and Discussion}

We verify the detection performance of the proposed algorithm by means of computer simulation and numerical calculation. In the simulation, for simplicity, we set the original PU signal as a random sparse signal obeying the Gauss distribution, which is sparse in the time domain. The degree of sparsity is $K=10 . \mathrm{SNR}=E / \sigma_{0}{ }^{2}$, where $E$ is the average power of $s(n)$. In the simulation, we assume that the total length of the signal $n=400$ and carry out 1000 times Monte Carlo simulation to get statistical average results.

It is assumed that there is no exchange of information between the CR nodes. That is, the detection results are independent of each other. For a single node user, we assume the detection probability of $P_{d}=0.9$ and $m_{i}=0.6$. Under different false-alarm probability $P_{f}$, the number of required observations for sequential compressed detection is shown in Figure 5.

The curves marked with "o"; " $\triangle$ "; and "+" correspond to simulation results when SNR is $21 \mathrm{~dB}, 24 \mathrm{~dB}$, and $27 \mathrm{~dB}$, respectively. It can be seen that the theoretical value is in good agreement with the simulation results under different SNRs. It can also be seen from Figure 5 that the number of observed values will be reduced with the increase of the SNR.

In the centralized collaborative sensing environment, Figure 6 shows, when $P_{d}=0.9$ and $m_{i}=0.6$, the required observations with NSCWSS and nonsequential compressed detection algorithm (CD) under different false-alarm probability. It can be seen from Figure 6 that the NSCWSS algorithm can reduce the number of observations from $35 \%$ to $50 \%$. However, since the DF strategy is adopted, there is no little variation in the average number of observations in a cooperative sensing environment compared to a single node.

Figure 7 is the number of sample values for $m_{i}=$ $\{0.5,1.0,1.5,2.0,2.5\}$ when $\mathrm{SNR}$ is from $15 \mathrm{~dB}$ to $25 \mathrm{~dB}$ in 


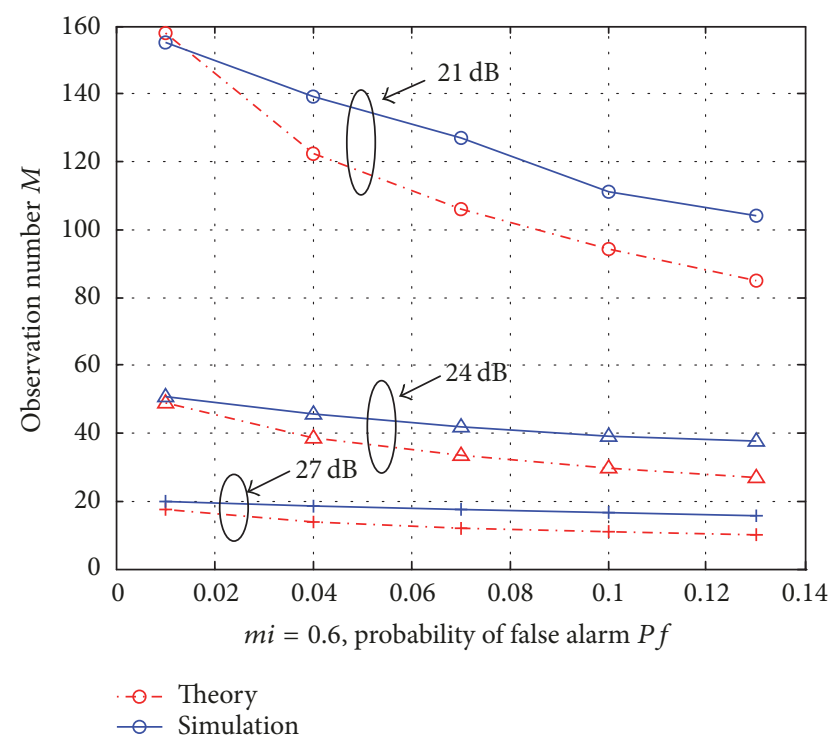

FIGURE 5: Observation number for different false-alarm probability.

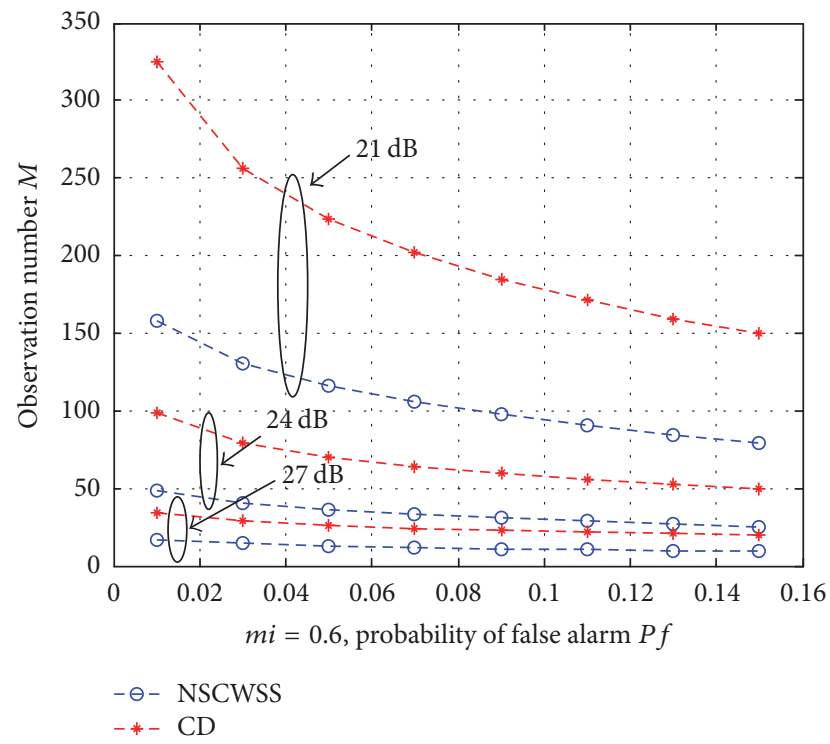

FIgURE 6: Performance of NSCWSS and CD at different SNR.

the collaborative sensing environment. We can see that the number of observations required for detection increases as the degree of channel fading increases.

In the case of Figures 8 and 9, there have been two malicious users. One of them implements an "Always-1" attack, and another one implements the "Always-0" attack. The reputation and weighted value of the malicious users are significantly reduced with the increase of number of sensing, which shows that algorithm can well identify malicious users to attack.

Assume that the number of $\mathrm{SU}$ is 50 . Figure 10 shows that NSCWSS algorithm has the better system sensing performance than "AND" algorithm, CD algorithm, and traditional WSPRT fusion algorithm with different proportion of malicious users under the "Always-1" or "Always-0" attack.

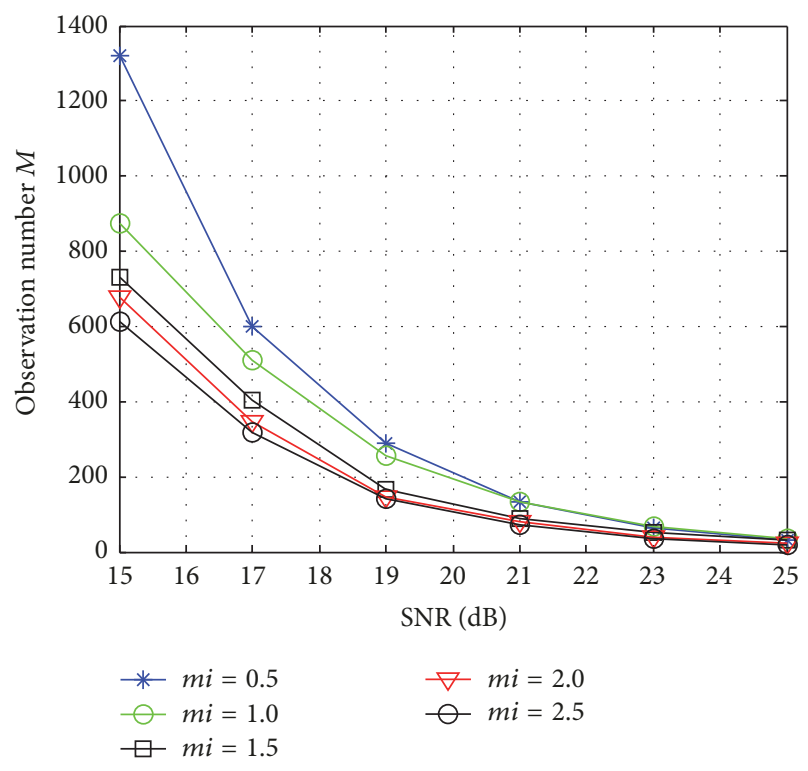

FIGURE 7: Required detection number $M$ versus SNR under different $m_{i}$.

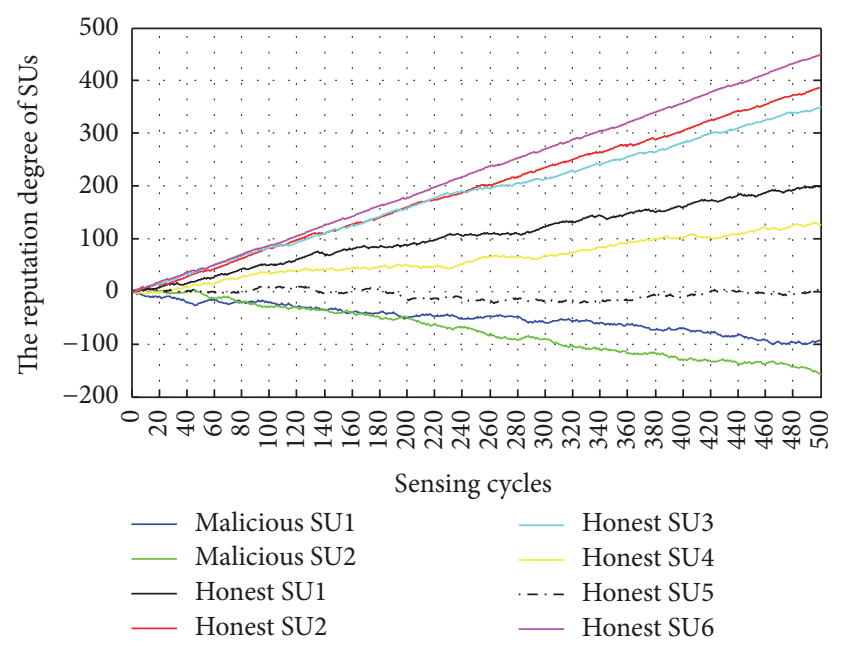

FIGURE 8: The changes of reputation for SUs.

Frome Figure 10, we can see that NSCWSS algorithm can maintain higher detection success probability, even if there have been more malicious users to attack.

\section{Conclusion}

In this paper, we propose a new robust weighted sequential nonreconstruction spectrum sensing algorithm based on history memory for wideband communications and analyze its detection performance. The simulation analysis shows that, under the prerequisite of guaranteeing certain detection probability, the proposed algorithm can greatly reduce the number of sampled observations and reduce the computation of sensing. Compared with the conventional sequential detection techniques, the proposed algorithm can better eliminate 


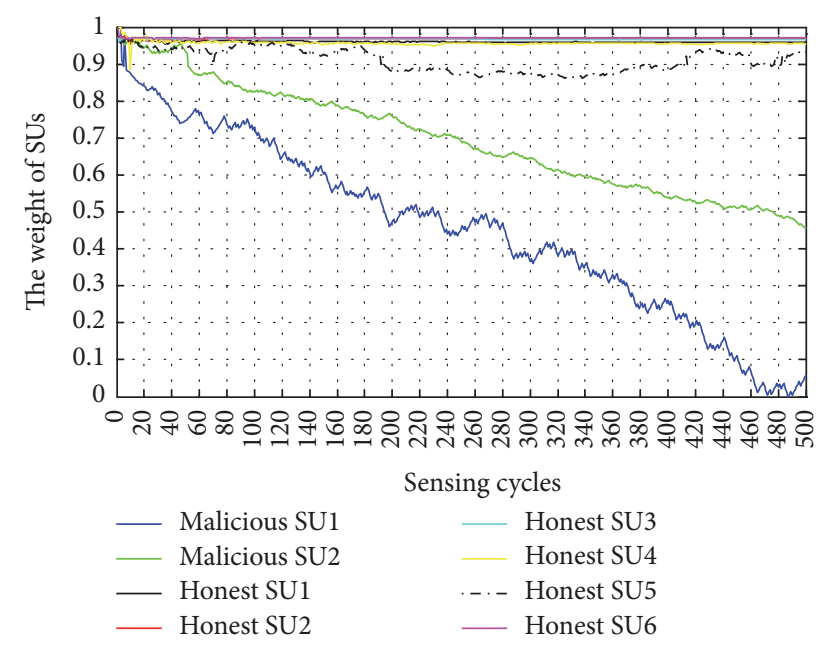

FIGURE 9: The changes of weighting for SUs.

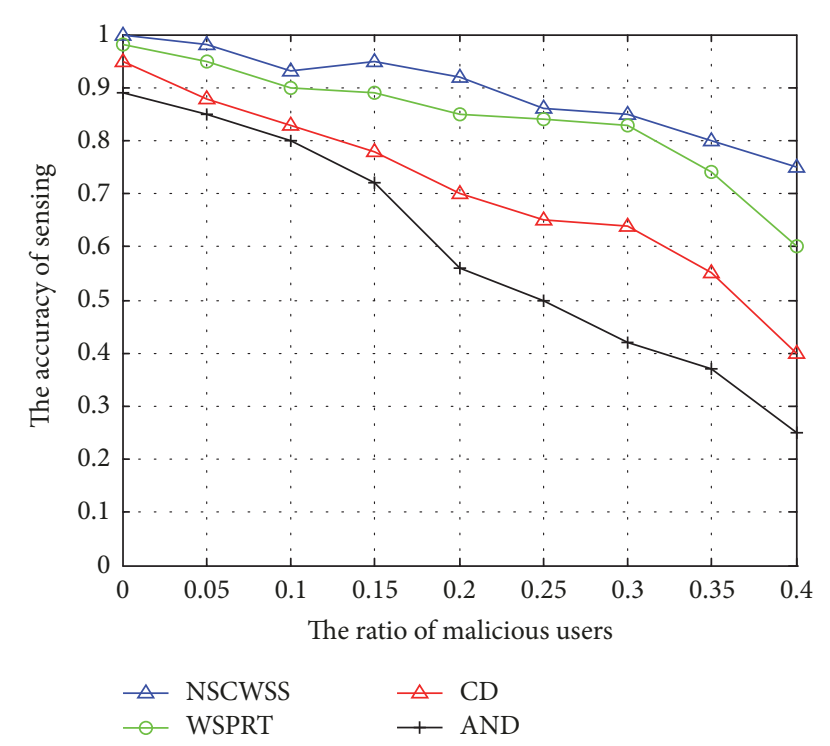

FIGURE 10: The performance analysis for different algrithms.

sample parameter uncertainty effect and improve the antiinterference ability of the SSDF attack, which can enhance the effectiveness and stability of the overall sensing of the spectrum, and it can satisfy the requirement of robustness for wideband spectrum sensing.

\section{Conflicts of Interest}

The authors declare that there are no conflicts of interest regarding the publication of this paper.

\section{Acknowledgments}

This project is supported by the National Natural Science Foundation of China (no. 61561017), the Hainan Province Natural Science Foundation of China (no. 617033), the Open Subproject of State Key Laboratory of Marine Resource Utilization in South China Sea (no. 2016013B), Oriented Project of State Key Laboratory of Marine Resource Utilization in South China Sea (no. DX2017012), the Postgraduate Practice and Innovation Project of Hainan University, and the Major Science and Technology Project of Hainan Province (no. ZDKJ2016015).

\section{References}

[1] H. Sun, A. Nallanathan, C. Wang, and Y. Chen, "Wideband spectrum sensing for cognitive radio networks: a survey," IEEE Wireless Communications Magazine, vol. 20, no. 2, pp. 74-81, 2013.

[2] G. I. Tsiropoulos, O. A. Dobre, M. H. Ahmed, and K. E. Baddour, "Radio resource allocation techniques for efficient spectrum access in cognitive radio networks," IEEE Communications Surveys \& Tutorials, vol. 18, no. 1, pp. 824-847, 2014.

[3] M. A. Davenport, P. T. Boufounos, M. B. Wakin, and R. G. Baraniuk, "Signal processing with compressive measurements," IEEE Journal of Selected Topics in Signal Processing, vol. 4, no. 2, pp. 445-460, 2010.

[4] Z. Tian and G. B. Giannakis, "Compressed sensing for wideband cognitive radios," in Proceedings of the IEEE International Conference on Acoustics, Speech and Signal Processing (ICASSP '07), pp. IV1357-IV1360, Honolulu, HI, USA, April 2007.

[5] J. Meng, W. Yin, H. Li, E. Hossain, and Z. Han, "Collaborative spectrum sensing from sparse observations in cognitive radio networks," IEEE Journal on Selected Areas in Communications, vol. 29, no. 2, pp. 327-337, 2011.

[6] S. Hong, "Multi-resolution bayesian compressive sensing for cognitive radio primary user detection," in Proceedings of the 53rd IEEE Global Communications Conference (GLOBECOM '10), pp. 1-6, December 2010.

[7] B. W. Li, Y. G. Li, and Y. G. Zhu, "Non-reconstruction compressive detection of random signal using maximum likelihood criterion and its analysis," Journal of Signal Processing, vol. 29, no. 8, pp. 996-1002, 2013.

[8] K.-T. Cao, X.-Q. Gao, and D.-L. Wang, "Wideband compressive spectrum sensing without reconstruction based on random matrix theory," Journal of Electronics and Information Technology, vol. 36, no. 12, pp. 2828-2834, 2014.

[9] D. M. Malioutov, S. R. Sanghavi, and A. S. Willsky, "Sequential compressed sensing," IEEE Journal of Selected Topics in Signal Processing, vol. 4, no. 2, pp. 435-444, 2010.

[10] H. Zheng, S. Xiao, and X. Wang, "Sequential compressive target detection in wireless sensor networks," in Proceedings of the 2011 IEEE International Conference on Communications (ICC '11), vol. 6, pp. 1-5, June 2011.

[11] L. Lu, X. Zhou, and G. Y. Li, "Optimal sequential detection in cognitive radio networks," in Proceedings of the 2012 IEEE Wireless Communications and Networking Conference (WCNC '12), pp. 289-293, April 2012.

[12] S. Y. Tu, X. Q. Song, Y. G. Zhu et al., “"Detection of random signal based on unreconstructed sequential compressive sensing and its analysis in cognitive wireless network," Journal of Signal Processing, vol. 30, no. 2, pp. 205-213, 2014.

[13] S. Althunibat, B. J. Denise, and F. Granelli, "Identification and punishment policies for spectrum sensing data falsification attackers using delivery-based assessment," IEEE Transactions on Vehicular Technology, vol. 65, no. 9, pp. 7308-7321, 2016.

[14] M. Nakagami, "The m-distribution-A general formula of intensity distribution of rapid fading," Statistical Methods in Radio Wave Propagation, pp. 3-34, 1960. 
[15] M. Abdel-Hafez and M. Cafak, "Performance analysis of digital cellular radio systems in Nakagami fading and correlated shadowing environment," IEEE Transactions on Vehicular Technology, vol. 48, no. 5, pp. 1381-1391, 1999.

[16] S. Hussain and X. N. Fernando, "Performance analysis of relaybased cooperative spectrum sensing in cognitive radio networks over non-identical nakagami-m channels," IEEE Transactions on Communications, vol. 62, no. 8, pp. 2733-2746, 2014.

[17] S. Hussain and X. N. Fernando, "Closed-form analysis of relay-based cognitive radio networks over Nakagami-m fading channels," IEEE Transactions on Vehicular Technology, vol. 63, no. 3, pp. 1193-1203, 2014.

[18] N. Kundargi and A. Tewfik, "A performance study of novel sequential energy detection methods for spectrum sensing," in Proceedings of the 2010 IEEE International Conference on Acoustics, Speech, and Signal Processing (ICASSP '10), pp. 30903093, Dallas, TX, USA, March 2010.

[19] A. Attar, H. Tang, A. V. Vasilakos, F. R. Yu, and V. C. M. Leung, "A survey of security challenges in cognitive radio networks: solutions and future research directions," Proceedings of the IEEE, vol. 100, no. 12, pp. 3172-3186, 2012.

[20] R. Chen, J.-M. Park, and K. Bian, "Robust distributed spectrum sensing in cognitive radio networks," in Proceedings of the 27th Conference on Computer Communications (INFOCOM '08), pp. 31-35, April 2008.

[21] J.-H. Zhao, F. Li, and T. Yang, "Weight sequential log-likelihood ratio detect algorithm with malicious users removing," Journal of China Universities of Posts and Telecommunications, vol. 20, no. 2, pp. 60-65, 2013. 


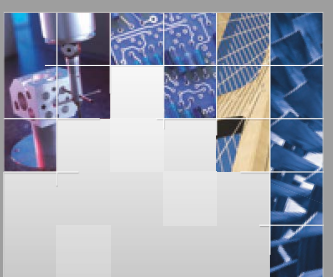

\section{Enfincering}
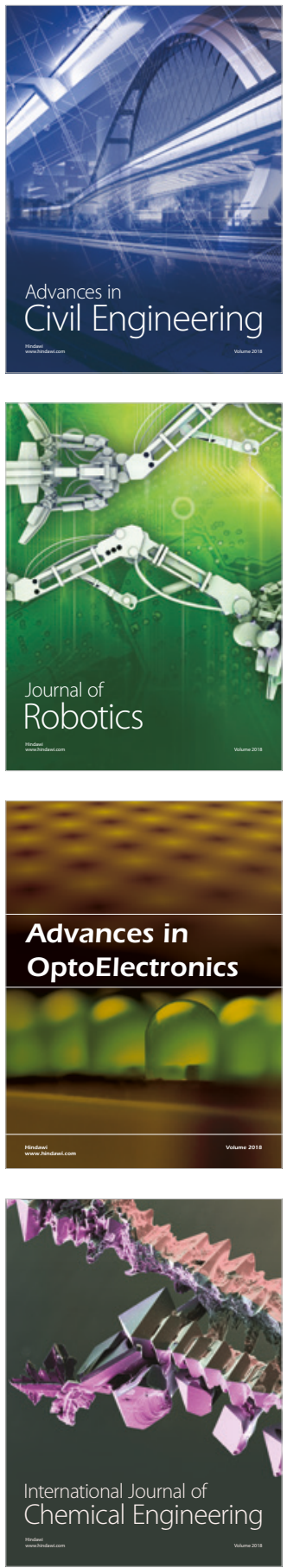

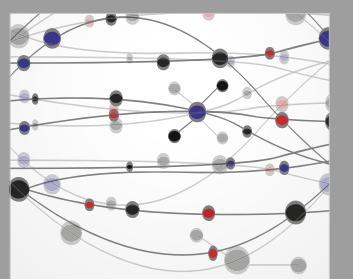

\section{Rotating \\ Machinery}

The Scientific World Journal

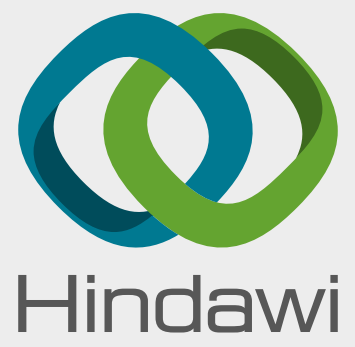

Submit your manuscripts at

www.hindawi.com
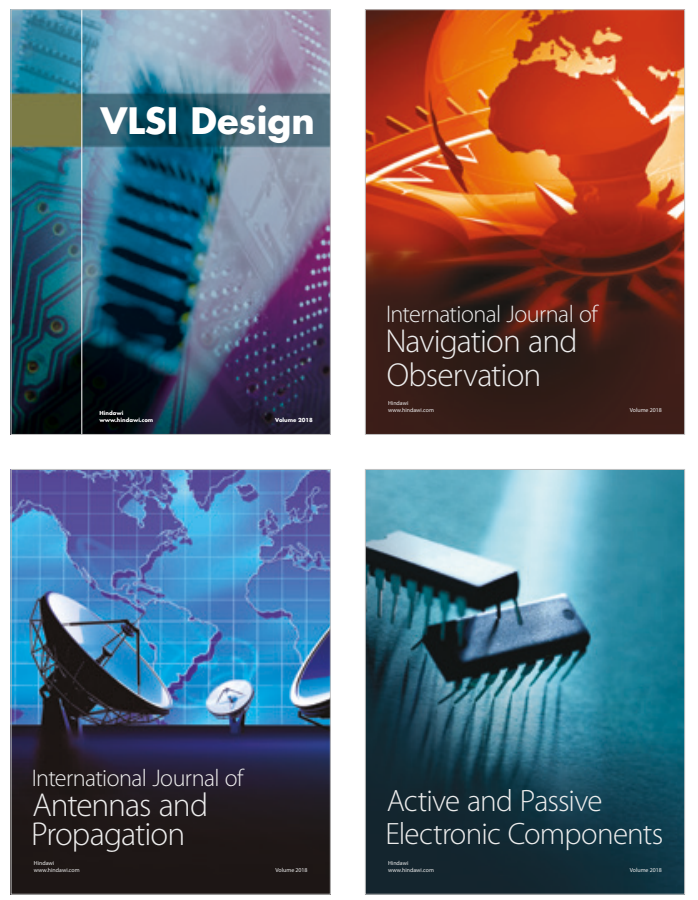
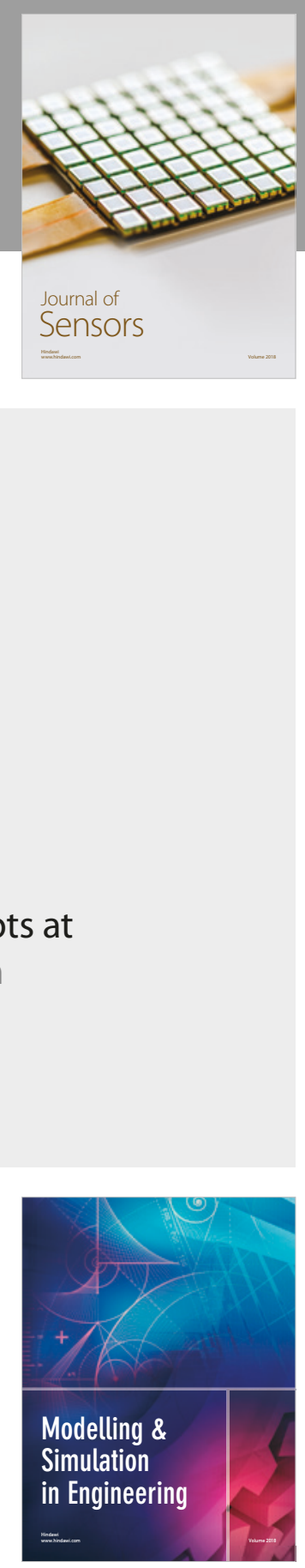

\section{Advances \\ Multimedia}
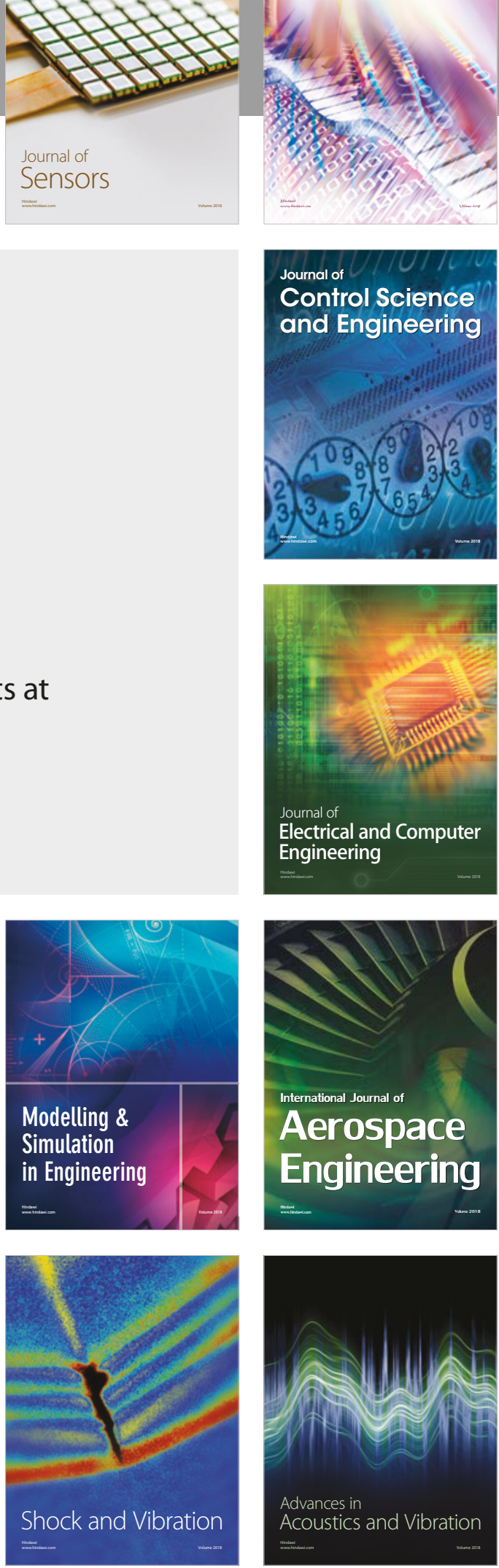